\title{
Dynamic simulation analysis of CT26 operating mechanism of high-voltage circuit breaker based on ADAMS
}

\author{
Kun Wang ${ }^{\mathrm{a}}$, Guoqing Chen, Shuting Wan and Pei Tian \\ North China Electric Power University, Baoding, P.R. China
}

\begin{abstract}
The reliability of high-voltage circuit breaker operating mechanism is important to ensure stable operation of the circuit breaker. The dynamic simulation model of CT26 spring operating mechanism is established using software ADAMS, and the dynamic simulation analysis of the feed motion of the operation mechanism is carried out, then the model is verified by contrasting the experimental data. The influence of the spring stiffness coefficient and friction to dynamic characteristics of circuit breaker are simulated and analyzed emphatically. Simulation results show that the breaking and closing time and the speed of actuator, the service life of the cam mechanism are directly affected by the above two factors. The simulation results and methods in this paper can provide reference for the research and daily maintenance of the circuit breaker operating mechanism.
\end{abstract}

Keywords: high-voltage circuit breaker; spring operation mechanism; dynamic simulation; Automatic Dynamic Analysis of Mechanical Systems(ADAMS).

\section{Introduction}

High voltage circuit breaker has been widely used in electric power system. The performance of circuit breaker directly affects the safe and stable operation of power distribution circuit and the downstream electric equipment, but the operating reliability of the circuit breaker operating mechanism is still very difficult to be satisfied. But the operational reliability of the circuit breaker operating mechanism is still not satisfactory. According to the investigation statistics of circuit breaker accident in China by State Grid and the Ministry of Electric Power, operating mechanism fault accounted for $66.4 \%$ of all failures, and the mechanical failure of the operating mechanism accounted for $55 \%$ of all failures. Mechanical failure is mainly malfunction of action failure such as ail-to-break and close, and malfunction. It follows that the reliability of the circuit breaker depends largely on its mechanical reliability. Therefore, it is important to improve the reliability of the operating mechanism.

Dynamic characteristic of the operating mechanism is an important factor to the performance of circuit breaker. Because of the more parts of the operating mechanism, the structure is complex, and the movement relationship is, the theoretical analysis is more difficult. Due to complicated structures and the various movement relationship among components between, theoretical analysis is difficult. So we can establish the virtual prototype model of the actuator by multi-body dynamics simulation software ADAMS, and conduct the simulation analysis on the dynamic characteristics of the breaking and closing course which can significantly shorten the development cycle and reduce the high cost of

a Corresponding author: wangkun9205@163.com

(C) 2016. The authors - Published by Atlantis Press 
physical prototype test. At present, the analysis of the dynamic characteristics of the operating mechanism has become an important research topic. In [3-5],the dynamic simulation model of circuit breaker operating mechanism was established, and the influence of the friction and the position of the baffle plate on the dynamic performance of the circuit breaker is studied. In [10-12],the dynamic change of contact force between cam and roller mechanism was studied. In [13-16], the dynamic simulation and optimization design of the connecting rod mechanism, the oil buffer and spring of the spring operating mechanism are carried out by using ADAMS.

In this paper, the influence of the spring stiffness coefficient and friction to dynamic characteristics of circuit breaker are simulated and analyzed emphatically based on the CT26 high voltage circuit breaker spring operating mechanism. The simulation results and methods in this paper can provide reference for the research and daily maintenance of the circuit breaker operating mechanism.

\section{ADAMS multi-body dynamics theory}

\subsection{The coordinate system and degree of freedom}

Mechanical system is divided into four components by simulation software ADAMS: part, constraint, force as well as the user defined algebraic and differential equation.

There are two coordinate systems in ADAMS/solver system: global coordinate and local coordinate. The global coordinate system is fixed in the ground reference. In ADAMS/Solver, we need to refer to the global coordinate system to create the geometry of the body coordinate system. The local coordinate system is used to locate the position of the key points and axes in the model, the body coordinate system and the mark point are the two major types of local coordinate system.

Degree of Freedom(DOF) in ADAMS is used to indicate the independent movement parameters number that the has definite movement, its formula is as follow:

$$
D O F=6 \times(n-1)-\sum_{i} n_{i}
$$

In Eq.(1), the $n$ indicates the number of components in the system (including the ground) and the $n_{i}$ indicates the number of DOF of each constraint in the system

\subsection{Multi rigid body dynamic analysis equation}

\subsubsection{Experimental measurement of the closing and breaking course}

To The Descartes coordinate $(p)$ of rigid body and Euler angle $(\varepsilon)$ which reflect the direction of rigid body are taken as generalized coordinate in Adams, that is $\mathrm{p}=[x, y, z] \mathrm{T}, \varepsilon=[\psi, \phi, \theta] \mathrm{T}$.

As a result, the generalized coordinate of the rigid body in ADAMS is expressed as follow.

$$
q_{i}=\left[\begin{array}{l}
p_{i} \\
\varepsilon_{i}
\end{array}\right]
$$

Based on the above established coordinates, the linear velocity and angular velocity of the rigid body are expressed as follow.

$$
\left\{\begin{array}{l}
u=\dot{q} \\
\omega=J \dot{\varepsilon}
\end{array}\right.
$$


In Eq.(3):

$$
J=\left[\begin{array}{ccc}
\sin \phi \sin \theta & 0 & \cos \phi \\
\cos \phi \sin \theta & 0 & -\sin \phi \\
\cos \theta & 1 & 0
\end{array}\right]
$$

The Dynamic equation of rigid body is as follow:

$$
k=\frac{1}{2} u^{T} M u+\frac{1}{2} \omega^{T} \bar{J}-
$$

In Eq.(5), the $M$ is Generalized mass matrix, $J$ is Generalized moment of inertia matrix, $K$ is kinetic energy.

\subsubsection{Experimental measurement of the closing and breaking course}

By consulting the books, the following second order differential equation is derived from the Lagrange equations of motion.

$$
\frac{d}{d t}\left[\frac{\partial K}{\partial q}\right]-\left(\frac{\partial k}{\partial q}\right)^{T}+\Phi_{q}^{T} \lambda=Q
$$

We select the ADAMS coordinates as the generalized coordinates, the formula (6) turned into as follow:

$$
\frac{d}{d t}\left[\begin{array}{l}
\left(\frac{\partial K}{\partial u}\right)^{T} \\
\left(\frac{\partial K}{\partial \zeta}\right)^{T}
\end{array}\right]-\left[\begin{array}{l}
\left(\frac{\partial K}{\partial p}\right)^{T} \\
\left(\frac{\partial K}{\partial \varepsilon}\right)^{T}
\end{array}\right]+\left[\begin{array}{l}
\Phi_{P}^{T} \lambda \\
\Phi_{\varepsilon}^{T} \lambda
\end{array}\right]=\left[\begin{array}{l}
\left(\Pi^{P}\right)^{T} f \\
\left(\Pi^{R}\right)^{T} \frac{\mathrm{n}}{2}
\end{array}\right]
$$

In Eq.(7):

$$
\left\{\begin{array}{c}
\frac{d}{d t}\left(\frac{\partial K}{\partial u}\right)^{T}=M u \\
\left(\frac{\partial K}{\partial p}\right)^{T}=0 \\
\Gamma=\frac{\partial K}{\partial \zeta}=B^{T} J B \zeta
\end{array}\right.
$$

So we can get the following equations of motion as follow.

$$
\left\{\begin{array}{c}
M u+\Phi_{p}^{\Gamma} \lambda=\left(\Pi^{p}\right)^{T} f \\
\Gamma-\frac{\partial K}{\partial \varepsilon}+\Phi_{s}^{T} \lambda=\left(\Pi^{R}\right)^{T} n
\end{array}\right.
$$

Finally, we linked up variation of generalized coordinates with time and linear as well as the angular momentum by differential equations of motion. Thus we obtained 15 equations to solve the dynamic numerical solution of the mechanical system. These equations are as follows: 


$$
\left\{\begin{array}{l}
M \dot{u}+\Phi_{p}^{T} \lambda-\left(\Pi^{p}\right)^{T} f=0 \\
\Gamma-B^{T} J B \zeta=0 \\
\dot{\Gamma}-\frac{\partial K}{\partial \varepsilon}+\Phi_{\delta}^{\Gamma} \lambda-\left(\Pi^{R}\right)^{T}-\overline{\mathrm{n}}=0 \\
\dot{p}-u=0 \\
\dot{\varepsilon}-\zeta=0
\end{array}\right.
$$

\section{The establishment and verification of dynamic model of operating mechanism}

\subsection{The establishment of dynamic simulation model}

To establish the dynamic model of the operating mechanism, we need to build the three-dimensional solid model,we established the 3D model of the CT26 spring operation mechanism with the modeling software Pro/E according to the drawings provided by the circuit breaker manufacturer, as shown in Figure 1.

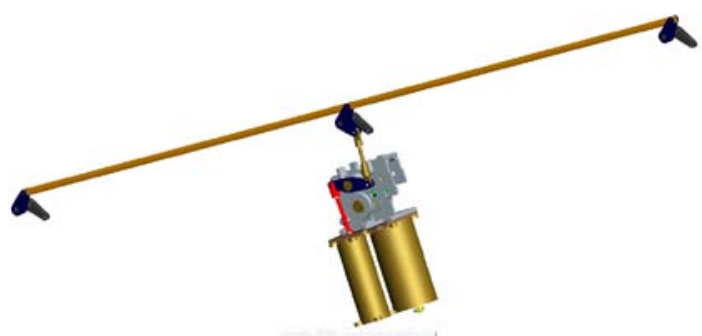

Figure1. 3D solid model

Because more components of the circuit breaker operating mechanism will increase the complexity of dynamic simulation, taking into account the simulation only focus on the dynamic relationship between the main bodies in the breaking and closing course, non-standard parts and other some unnecessary parts of the simulation process was simplified such as the pawl, spring outer sleevet, connecting fasteners and standard parts. The main structure of the simplified three- dimensional model is shown in Figure 2.
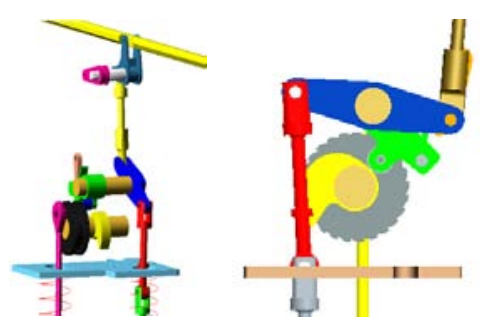

Figure 2. Simplified model

The dynamic simulation model can be built after the 3D model is established. Graphical data exchange between Pro/E and ADAMS generally uses the Parasolid standard. It will import into the ADAMS after the 3D model file is stored as Parasolid standard file format $\left({ }^{*} \mathrm{x} \_t\right)$, then we still need to set up a variety of parameters for the imported model, it mainly includes:

1) Setting up the environment unit for MMKS (mm, kg, N) form;

2) Adding material properties for components

3) Adding motion pair constraints:adding fixed vice, revolute vice, planar vice, etc according to 
the assembly and movement relationship between all parts;

4) Adding spring force and contact force:In the operating mechanism, the closing and breaking spring are replaced by the spring dampers. the parameters as shown in Figure 3, The pre tightening force is achieved by changing the length of the initial spring. Pre-tightening force is realized by changing the initial length of spring. The collision is replaced by the addition of contact force in the process of mechanism movement, mainly includes the contact force between the cam and roller, the latch and the roller support and other components.

The dynamic simulation model is shown in Figure 4 after all of parameters setting finished.

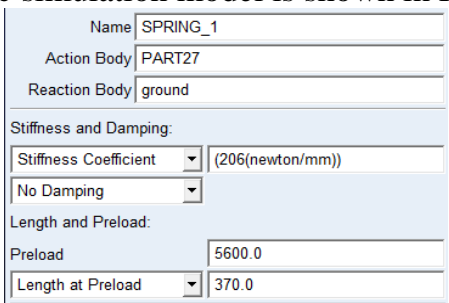

(a) The closing spring parameters

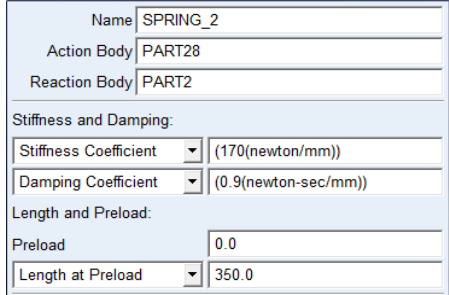

(b) The breaking spring parameters

Figure 3. Spring parameters settings
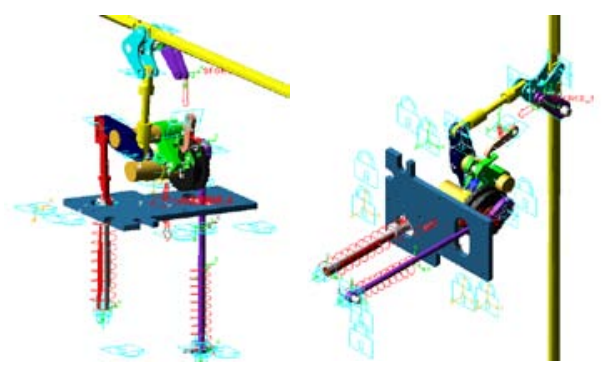

Figure 4. Dynamic simulation model

\subsection{Verification of the simulation models}

\subsubsection{Experimental measurement of the closing and breaking course}

In order to verify the correctness of the dynamic simulation model, it is necesary to make a comparative analysis of the experimental data and the simulation results. Therefore we measured dynamic characteristic parameters of the moving contact in closing and breaking course of circuit breaker mechanism by using the high voltage switch tester, includes the length - time and velocity time curve of the moving contact, and the experimental measurement results are shown in figure 5.

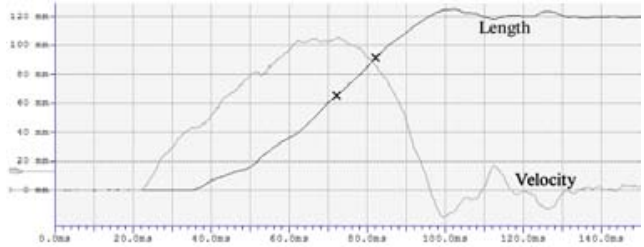

(a) The dynamic characteristics of the closing course

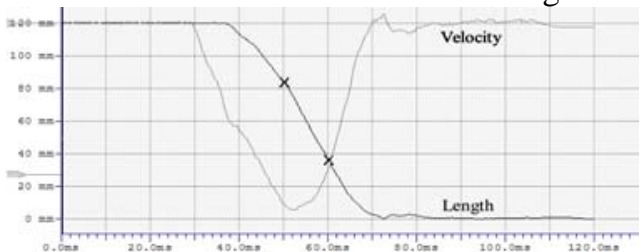

(b)The dynamic characteristics of the breaking course

Figure 5. The experimental measured movement characteristics of the breaking and closing course

The dynamic characteristics curve of the closing course are shown in figure 5(a). The time range of the moving contact movement is $37 \sim 106 \mathrm{~ms}$, the total time is about $69 \mathrm{~ms}$ and the maximum length is about $120 \mathrm{~mm}$. The dynamic characteristics curve of the breaking course are shown in figure 5(b). The time range of the moving contact movement is $49 \sim 86 \mathrm{~ms}$, the total time is about $37 \mathrm{~ms}$. 


\subsubsection{Dynamic simulation analysis of the closing course}

The principle of closing course of the circuit breaker operating mechanism is that the closing spring drives the cam to rotate, then the roller on the output crank arm of the cam is pressed to cause the crank arm to move upward. Finally through the connecting rod to drive the contact movement, finally the moving contact moved which was driven by the connecting rod. At the same time, the energy storage of the breaking spring is carried out by the compression of the breaking spring crank arm, prepare for the breaking operation.

The structure of the operating mechanism shows that the length of internal crank arm is the same as the moving contact in the vertical direction,so we choose the length and velocity data of the internal crank arm as the characteristic parameters of the moving contact. The dynamics simulation results of closing course are shown in Figure 6.

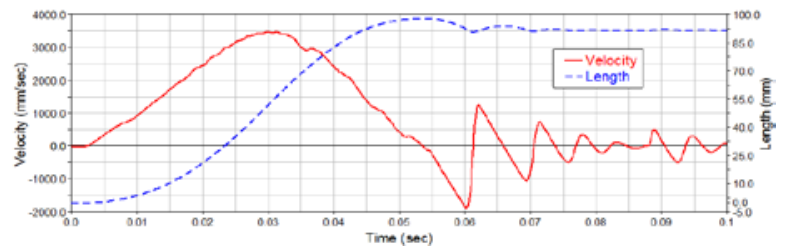

Figure 6. Simulation result of the closing course

We can know that the moving contact movement time is about 63ms in Figure 6, which is close to the experimental result. The maximum length is about $98 \mathrm{~mm}$. and the Peak velocity is $3500 \mathrm{~mm} / \mathrm{s}$, the length of the moving contact is slightly smaller than experimental result which is shown in figure 5(a).

\subsubsection{Dynamic simulation analysis of the breaking course}

The principle of breaking course of the circuit breaker operating mechanism is that the breaking spring releases its energy to drive the contact realizes the break-brake movement through the crank arm, the connecting rod. The breaking spring has completed the storage operation in the closing course. The dynamics simulation results of breaking course are shown in Figure 7.

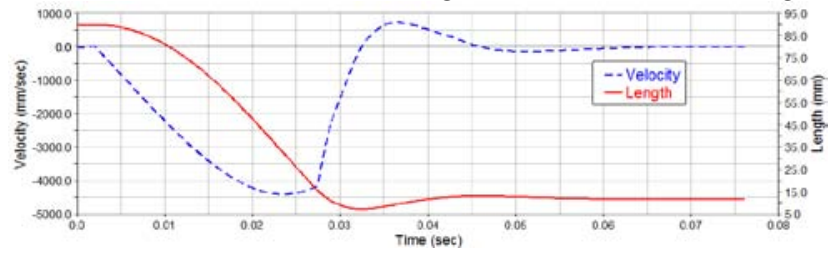

Figure 7. Simulation result of the breaking course

We can know that the moving contact movement time is about $34 \mathrm{~ms}$ in Figure 7, which is close to the experimental result and the break-brake time conform to the technical parameters( $35 \pm 7 \mathrm{~ms})$ of the operating mechanism. The peak velocity is $3500 \mathrm{~mm} / \mathrm{s}$ and the length of the moving contact is slightly smaller than experimental result which is shown in figure 5(b).

\subsubsection{Discussion}

The simulation results show that the dynamic simulation results are close to the experimental results. Movement tendency and curve shape of length-time and velocity-time curve are roughly anastomotic with the curves of the experimental measurement shown in Figure 5. So as to verify the accuracy of the established simulation model. 


\section{Influence of different spring stiffness on dynamic characteristics}

We can know from the operation principle of the operating mechanism that springs are the main power source for closing and breaking movement of operating mechanism. The spring stiffness directly affects the dynamic characteristics of the closing and breaking course. The influence of different stiffness on the moving contact length, velocity and the contact force between the cam and the roller were simulated, including normal stiffness, relatively small stiffness and relatively large stiffness those three operating conditions. Simulation results are shown in figure 8, 9 and 10.

\subsection{The influence of different spring stiffness on the movement characteristics}

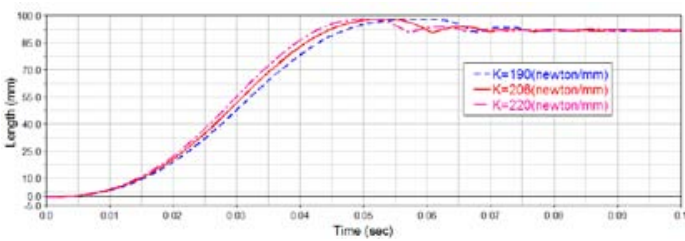

(a) Length of moving contact

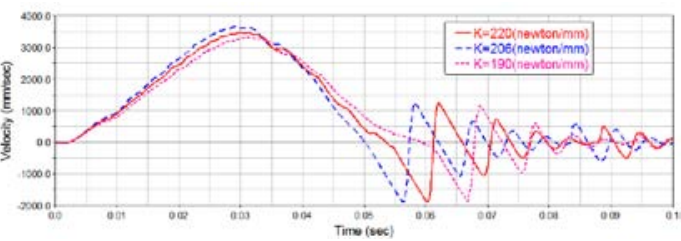

(b) Velocity of moving contact

Figure 8. Movement characteristics in different closing spring stiffness in closing course

The simulation results of contact movement characteristics in different closing spring stiffness in closing course are shown in Figure 8.

It can be seen from the figure that the moving contact length remains the same in different closing spring stiffness, but the moving contact movement time and velocity are affected. When the spring stiffness is relatively small, that is, $K=190 \mathrm{~N} / \mathrm{mm}$, moving contact movement period increased,peak velocity decreased from $3300 \mathrm{~mm} / \mathrm{s}$ to $3500 \mathrm{~mm} / \mathrm{s}$. When the spring stiffness is relatively large, that is, $\mathrm{K}=220 \mathrm{~N} / \mathrm{mm}$, moving contact movement period decreased, and the peak velocity increased from $3500 \mathrm{~mm} / \mathrm{s}$ to $3700 \mathrm{~mm} / \mathrm{s}$. On the whole, the tendency of the length and velocity remained the same.

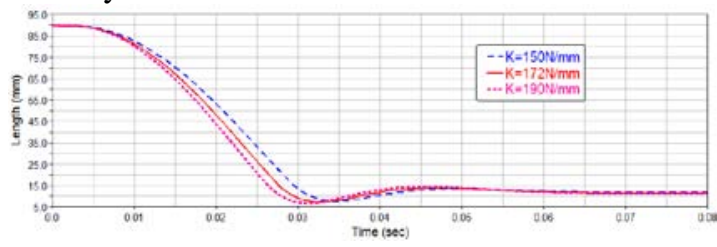

(a) Length of moving contact

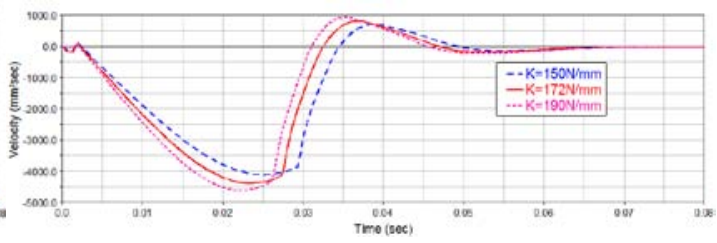

(b) Velocity of moving contact

Figure 9. Movement characteristics in different closing spring stiffness in breaking course

The simulation results of contact movement characteristics in different breaking spring stiffness in breaking course are shown in Figure 9.

It can be seen from the figure that the moving contact length remains the same in different breaking spring stiffness, but the moving contact movement time and velocity are affected. When the spring stiffness is relatively small, that is, $K=150 \mathrm{~N} / \mathrm{mm}$, moving contact movement period increased, peak velocity decreased from $4500 \mathrm{~mm} / \mathrm{s}$ to $4200 \mathrm{~mm} / \mathrm{s}$. When the spring stiffness is relatively large, that is, $\mathrm{K}=190 \mathrm{~N} / \mathrm{mm}$, moving contact movement period decreased, and the peak velocity increased from $4500 \mathrm{~mm} / \mathrm{s}$ to $4700 \mathrm{~mm} / \mathrm{s}$.

We can know from the function of the circuit breaker that it is used to switch on and off the circuit during normal operation, and could break the circuit rapidly when faults occurred, therefore, the breaking and closing time and contact movement velocity will directly affect the breaking and closing performance of circuit breaker. Only in ensuring the proper breaking and closing time, can the circuit breaker to give full play to its ability to switch on and off the electric current. If the closing and breaking time is too short, it will makes the circuit breaker will not be able to cut off the fault rapidly, because the circuit breaker arc insulation strength and arc extinguishing ability is not enough, and 
eventually lead to rest riking accident. If the closing and breaking time is too long, the operating mechanism is unable to remove the fault in time, causing arcing time increase, may eventually makes the contact burning or explosion.

\subsection{Influence of different spring stiffness on the contact force between the cam and the roller}

The cam and the roller will impact repeatedly in the closing course, the impact contact force is large with a wide fluctuation. The needle roller bearing has become the most vulnerable parts due to impact force, therefore it is very necessary to study the change of contact force between the cam and the roller. The simulation results of contact force changes between the cam and the roller in different stiffness in closing course are shown in Figure 10.

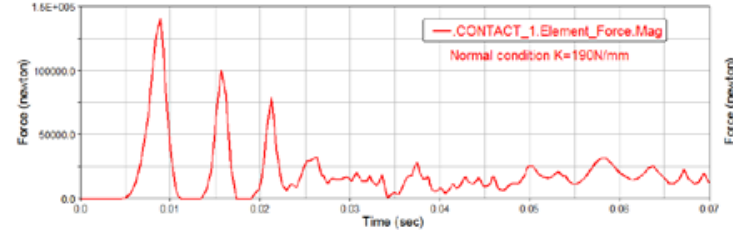

(a) relatively small stiffness: $\mathrm{K}=190 \mathrm{~N} / \mathrm{mm}$

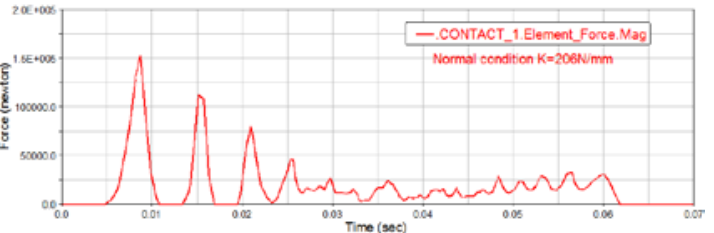

(b) normal stiffness: $\mathrm{K}=206 \mathrm{~N} / \mathrm{mm}$

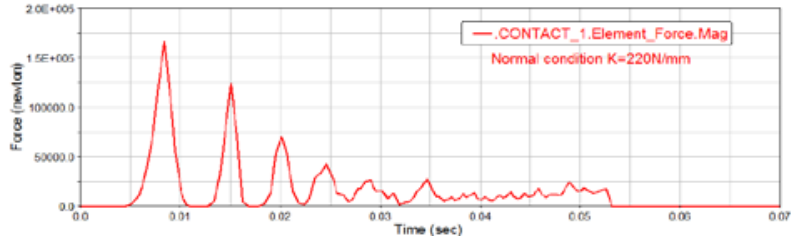

(c) relatively large stiffness: $\mathrm{K}=220 \mathrm{~N} / \mathrm{mm}$

Figure 10. The contact force between the cam and roller in different closing spring stiffness

We can know from the simulation results shown in Figure 10 that the contact force between the cam and the roller in closing course is indeed relatively large, Multiple peaks in the curve are also demonstrated that there are many impact collision between the two parts, and the maximum contact force is also increased with the increase of the spring stiffness. The simulation results are consistent with the theoretical analysis and inference. Excessive force and repeated impact will aggravate the damage of the contact surface of the cam and roller, and cause the fracture of the needle rollers of bearing and the deformation of bearing outer ring, furthermore it can also cause large vibration and noise of the whole circuit breaker mechanism.

\subsection{Discussion}

We can know from the above analysis that stiffness of the spring has a great influence on the dynamic characteristics of the circuit breaker. This puts forward higher requirements on the level of circuit breaker manufacturing and assembly, the components of Cam mechanism should be pay attention to improve the accuracy and machining in the design and manufacturing process to ensure the cam mechanism and needle roller bearings have higher hardness and fatigue strength. In addition, circuit breaker exercise dynamic mechanism should be carefully calibrated closing and braking spring in the assembly process to make the closing and breaking time of operating mechanism and other characteristics could meet the technical requirements of the product, so as to ensure the operation of circuit breaker steadily and normally.

\section{Influence of different friction coefficient on dynamic characteristics}

The friction force is an important factor to affect the performance of the machine in mechanical 
movement process. Harmful friction will cause the kinematic pairs suffer abrasion and reduce the strength and reliability of components. It can still cause the kinematic pairs suffer heat expansion in serious case which will lead to kinematic pairs running sluggish, even stuck fault.

The influence of different friction coefficient of the cam, roller and link mechanism on dynamic characteristics in closing course was simulated, including normal stiffness $(\mu=0.02)$, friction coefficient of $0.1(\mu=0.1)$ and friction coefficient of $0.12(\mu=0.12)$ those three operating conditions. Simulation results are shown in figure 11.

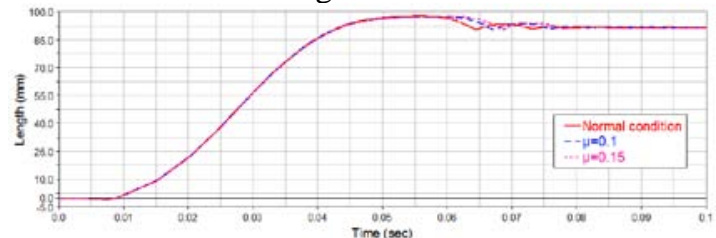

(a) Length of moving contact

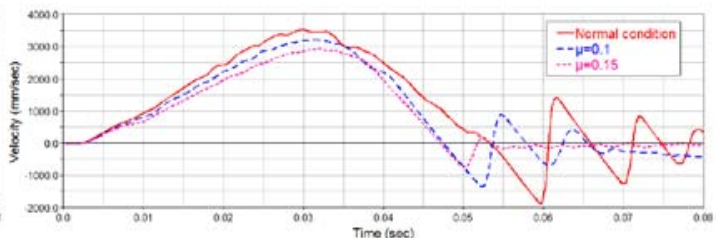

(b) Velocity of moving contact

Figure 11. Movement characteristics under different friction condictions in closing course

According to figure 11 , when the friction coefficient increases to $0.1(\mu=0.1)$, moving contact movement period increased from $63 \mathrm{~ms}$ in the normal state to $67 \mathrm{~ms}$, peak velocity decreased from $3500 \mathrm{~mm} / \mathrm{s}$ to $3200 \mathrm{~mm} / \mathrm{s}$, and the oscillatory convergence time of the velocity reduced at the end of the closing course. When the friction coefficient continues increase to $0.12(\mu=0.12)$, moving contact movement period increased to $69 \mathrm{~ms}$, the peak velocity decreased to $2900 \mathrm{~mm} / \mathrm{s}$. We deduce that as the friction coefficient continues to increase, the entire mechanism is likely to be stucked when the friction coefficient beyond a definite condition.

The simulation analysis shows that the friction of the system has a great influence on the dynamic characteristics of the circuit breaker. With the increase of switching operation times, the decrease of lubrication will lead to the increase of friction force, excessive friction can even cause the refusing open and refusing close fault of the operating mechanism. So we need to strengthen the surveillance of the circuit breaker in the operation and energize daily lubricating maintenance of the operating mechanism to ensure the normal operation of the operating mechanism.

\section{Conclusions}

The dynamic simulation model of CT26 spring operating mechanism is established using software ADAMS, and the dynamic simulation analysis of the feed motion of the operation mechanism is carried out. The following conclusions can be drawn from the analysis of the simulation results:

(1)The spring stiffness is relatively small, moving contact movement period will increase and the peak velocity decrease.This will makes the circuit breaker will not be able to cut off the fault rapidly.

(2)The contact force between the cam and the roller in closing course will increase with the increase of the spring stiffness, it directly affected the service life of the cam mechanism.

(3)The increase of friction coefficient of mechanism will cause the closing and breaking period increase and the peak velocity decrease. Excessive friction can even cause the refusing open and refusing close fault of the operating mechanism

The simulation results and methods can provide reference for the research and daily maintenance of the circuit breaker operating mechanism.

\section{References}

1. D. X.TAN, Y. LI, J. Y. XU. R\&D of Permanent Magnetism Operating Mechanism for 35kV Vacuum Circuit Breaker [J]. Electrical Equipment, 6, 2: 26-28, (2005).

2. Z. W. MA, Y. TU. Dynamic Simulation and Stress Analysis of DC Low Voltage Circuit Breaker Considering Electrodynamic Force [J]. Low Voltage Apparatus, 6: 10-14, (2013). 
3. T. AN, Q. F. WANG, J. Z. TANG, etc. Application of CB operating mechanism simulation model in fault diagnosis [J]. Advanced Technology of Electrical Engineering and Energy, 29, 1: 41-44, (2010).

4. H. X. MIAO, H. H. WANG, B. S. QI. Research on dynamic modeling and simulating of operating mechanism of high-voltage circuit breaker [J]. Computer Engineering and Applications, 46, 17: 212-215, (2010).

5. Y. X. LI, X. LIN, J. Y. XU, etc. Design of a novel permanent magnet brushless DC motor-driven operating mechanism for high-voltage circuit breaker and its dynamic simulation [J]. Power System Technology, 34, 1: 185-189, (2010).

6. R. S. Shen,Y. Liu, H. Xu, etc. Analysis on Error of Switch Action Speed Measurement in Circuit Breaker Mechanical Characteristics Test [J]. Hebei Electric Power, 6: 23-25, (2015).

7. X. LIN, L. F. SONG, Y. X. LI, etc. Multi-body dynamics simulation and analysis on novel motor -operating mechanism for vacuum circuit breakers[J]. Power System Technology, 36, 3: 76-80, (2012).

8. Z. J. WANG, X. X. JIANG, C. X. BAI, etc. Dynamic Simulation of contact impact of high voltage circuit breaker [J]. Electrical Manufacturing, 8: 42-49, (2014)

9. W. YANG, M. Z. RONG, X. H. WANG, etc. Dynamic simulation of high-voltage circuit breaker concerning electrodynamic force [J]. Proceedings of the CSEE, 23, 5: 103-107, (2003).

10. W. YANG, M. Z. RONG, D. G. CHEN, etc. Optimum design of high-voltage circuit breakers based on mechanism dynamic features simulation [J]. Journal of Xi'an Jiaotong University, 36, 12: 1211-1214, (2002).

11. S. Y. LI, R. B. DENG. Dynamic simulation and application of colliding process of cam and rolling bearing in circuit breaker[J]. High Voltage Apparatus, 44, 6: 497-500, (2008).

12. R. B. DENG. Dynamic simulation and secondary development of cam collision in high-voltage switch spring operating mechanism [D]. Kunming:Kunming University of Science and Technology, (2005).

13. X. F. Zhang. Base on Virtual Prototyping Technology Mehanism Simulation of Circuit Break and Optimization Mechanism Sping [D]. Soochow University, (2009).

14. Y. Z. ZHANG, K. X. XU, C. Y. YAN, etc. Optimization and simulation of the spring actuator for circuit breakers [J]. High Voltage Apparatus, 50, 4: 66-71, (2014).

15. J. S. Zhang, D. G. Chen, H. W. Liu. Dynamic simulation and optimum design of low-voltage circuit breaker[J]. Proceedings of the CSEE, 24, 3: 102-208, (2004).

16. Y. B. CUI, Z. GAO, Y. P. CAO. Dynamics simulation study on application ADAMS for the operating device for HV circuit [J]. Machinery Design and Manufacture, 4: 66-67, (2006). 